\title{
Dyadotropic Polynomials. II
}

\author{
By Harvey Cohn
}

\begin{abstract}
A computer search is made to test whether independent units of dyadotropic biquadratic fields (constructed parametrically) are always fundamental. A few exceptions are found. Because of the exponential rate of growth of parameters, $p$-adic methods are desirable. The feasibility of dyadotropic normed polynomials is also considered for general cases of degree 2 and special cases of degree 6.
\end{abstract}

7. Introduction. We shall ask if units given (largely) by a parametrized formula (in Section 9 below) are always fundamental.

To take a very familiar analogy, consider the real quadratic field $k_{2}=\mathbf{Q}\left(d^{1 / 2}\right)$ with $d=m^{2} \pm 4, m$ a positive integer. Take $\epsilon_{1}=\left(m+d^{1 / 2}\right) / 2$ the "obvious" unit. It can scarcely be expected to be "generally" fundamental. For example, if $\epsilon_{1}$ should be fundamental, then $\epsilon_{1}^{g}=\left(m_{g}+n_{g} d^{1 / 2}\right) / 2$, which corresponds (say for $g$ odd) to $m_{g}^{2}-n_{g}^{2} d=\mp 4$. So for $m=m_{g}, d_{g}=m_{g}^{2} \pm 4$ leads to $\epsilon_{1}^{g}$. The situation can be improved (for the fundamentality of $\epsilon_{1}$ ) if we require that $d$ be fundamental. We could also avoid having $\epsilon_{1}$ a perfect square by choosing the sign so that $N \epsilon_{1}=-1$, but aside from such devices, we should expect a "free" parameter to produce occasional nonfundamental units. Our parameters here are not so "free", however, because of the parametrization procedures.

We are about to test 84 cases within the reasonable bounds of single-integral and double-decimal arithmetic, and we find surprisingly few cases where the units, as parametrized, fail to be fundamental. They are shown in Table III (see Section 11 below), and they leave us with the conclusions (11.1) and the conjectures (11.2-3).

8. Local Search Method. We find it computationally convenient to search for $p$-fundamental units for primes $p \geqslant 2$. A set of units $U$ of a field $k$ is called " $p$-fundamental" if the multiplicative group $\langle U\rangle$ (generated by elements of $U$ ) contains no element of $k$ which is a $p$-power (to within a factor of a root of unity perhaps) except trivial ones, i.e., $p$-powers of elements of the group $\langle U\rangle$. Thus, a dependent set of units is never $p$-fundamental for any $p$, as the dependency relation is essentially a nontrivial one. Conversely, any set $U$ which is $p$-fundamental for all $p$ is fundamental provided it has the proper Dirichlet rank (see Section 5 above). The group of units of

Received May 24, 1977; revised January 24, 1978.

AMS (MOS) subject classifications (1970). Primary 12A45, 12A65; Secondary 10B10, $10 \mathrm{~B} 20$.

Key words and phrases. Biquadratic fields, units, machine computation.

* Research supported by NSF Grant MCS 76-06744. The sections, tables, and bibliographical items are numbered successively with "Part I" (item [12] of the bibliography). The author is indebted to the Mathematical Institute of the University of Copenhagen for support and usage of the UNIVAC 1100. 
$k$, of course, also require roots of unity (always \pm 1 in the present computation).

There are discriminant and regulator estimates which could limit the range of $p$ which one must test, and indeed such limits work well in the quadratic case, and some higher fields [14]. For exponentially growing parameters, however, those bounds are not helpful in view of the fact that the numerical feasibility of the search seems to weaken rapidly as $p$ grows.

The computational test consists of finding some well-chosen primes $q$ where the "Euler totient" $\phi(q) \equiv 0(\bmod p)$, and for which the rational prime $q$ splits completely so that all algebraic numbers are congruent to integers modulo a factor of $q$. Then we are testing ordinary integers for presence of $p$-powers and (hopefully) enough negative outcomes will establish $p$-fundamentality.

9. Main Results. To fix a more convenient notation, particularly for signs of units, we recapitulate some formulas of Section 3 (above). For a triple of integers, $(e, a, d),\left(|e|=2^{T_{0} / 2}\right)$ we define $\xi$ by $f(\xi)=0$, and with it certain fields:

$$
\begin{gathered}
f(x)=x^{4}+a x^{3}+\left(2 e+\left(a^{2}-d\right) / 4\right) x^{2}+a e x+e^{2}, \\
k_{4}=k_{2}(\xi) \supset k_{2}=\mathrm{Q}\left(d^{1 / 2}\right), \\
k_{8}=k_{4}\left(d^{1 / 2}\right), \quad d^{\prime}=\left(\left(a^{2}-d\right) / 8-2 e\right)^{2}-e d .
\end{gathered}
$$

Here $k_{8}$ is the normal closure of $k_{4}$. The values $(e, a, d)$ were denoted by $[2 ; u, v]$ and $[2 ; u, v, w]$ (respectively) and are

$$
\begin{aligned}
& e=2, \quad a=(-1)^{v}\left(2^{u}-(-1)^{u}\right) / 3, \quad d=(a+6)^{2}+(-1)^{u+v} 8 \\
& e=-2, \quad a=-(-1)^{w}\left(2^{u}+(-1)^{v}\right), \quad d=(a-2)^{2}-(-1)^{v+w} 8
\end{aligned}
$$

Here $u \geqslant 1$ and $v, w$ are 0 or 1 ; but we exclude $[-2 ; 1,0,1]$, where $d=9$. There are exceptional cases

$$
(e, a, d)=\begin{aligned}
& (4,1,113)_{3},(-4,5,-7)_{5},(-4,1,17)_{3}, \\
& (-4,3,17)_{4},(-4,1,33)_{2},(-4,-3,73)_{4} .
\end{aligned}
$$

Here the subscript denotes $T_{-1}$ where $|f(-1)|=2^{T}-1,(|f(1)|=2$ by contrast). For cases (9.3a, b), $T_{-1}=u+1$. In all but one case $k_{8} \supset k_{4}$ (strictly). The exception is $(-4,-3,73)$ where $d=73, d^{\prime}=4 d$ so $k_{4}=k_{8}$, the uniquely defined (abelian) biquadratic subfield of $\mathbf{Q}(\exp 2 \pi i / 73)$.

We rewrite some formulas for units in Section 4 to fix the signs (as we no longer deal only with absolute values!)

$$
\begin{gathered}
\left\{\begin{array}{cc}
\alpha=\left(a+d^{1 / 2}\right) / 2, & \mu=\alpha^{2}-4 e, \\
\alpha^{\prime}=\left(a-d^{1 / 2}\right) / 2, & \mu^{\prime}=\alpha^{\prime 2}-4 e,
\end{array}\right. \\
\left\{\begin{array}{cc}
\xi=\left(-\alpha+\mu^{1 / 2}\right) / 2, & \xi^{\prime}=\left(-\alpha^{\prime}+\mu^{\prime 1 / 2}\right) / 2, \\
S \xi=\left(-\alpha-\mu^{1 / 2}\right) / 2, & S \xi^{\prime}=\left(-\alpha^{\prime}-\mu^{\prime 1 / 2}\right) / 2,
\end{array}\right.
\end{gathered}
$$




$$
\begin{gathered}
\gamma_{1}=(1+e)+\alpha, \quad \gamma_{-1}=(1+e)-\alpha \\
\epsilon_{1}=\gamma_{1}^{T-1} / \gamma_{-1}= \pm \epsilon^{g}
\end{gathered}
$$

for $\epsilon$ the fundamental unit of $k_{2}(\epsilon>1)$ and $g \in \mathbf{Z}$. We now test the set of units

$$
\begin{aligned}
& U=\left\{\epsilon, \Omega_{1}, \Omega_{2}\right\}, \\
& \Omega_{1}=(\xi-1)^{T-1} /(\xi+1), \\
& \Omega_{2}=\left\{\begin{array}{l}
\xi^{2}(\xi-1)^{2} / 2 \gamma_{1}, e= \pm 2 \\
\xi(\xi-1)^{2} / 2 \gamma_{1}, e= \pm 4
\end{array}\right\} .
\end{aligned}
$$

Thus, the conjugates are related over $k_{2}$ by

$$
\begin{gathered}
\Omega_{1} S \Omega_{1}=\epsilon_{1}, \\
\Omega_{2} S \Omega_{2}=\left\{\begin{array}{l}
1, e= \pm 2 \\
\operatorname{sgn} e, e= \pm 4
\end{array}\right\} .
\end{gathered}
$$

The set $U$ is independent to the extent of Theorem 5.4 (above), in particular after the exclusion of cases where $k_{8}$ is not totally real. (See Table II above.)

For computational convenience, we define

$$
\Omega_{2}^{*}=\left\{\begin{array}{l}
\xi(\xi-1), e= \pm 2 \\
\xi(\xi-1)^{2}, e= \pm 4
\end{array}\right\},
$$

so we have the units (of $k_{4}$ )

$$
\Omega_{2} / S \Omega_{2}=\left\{\begin{array}{l}
\left(\Omega_{2}^{*} / S \Omega_{2}^{*}\right)^{2}, e= \pm 2 \\
\Omega_{2}^{*} / S \Omega_{2}^{*}, e= \pm 4
\end{array}\right\} .
$$

THEOREM 9.12. For $e= \pm 2$ the units $\left\{\epsilon, \Omega_{1}, \Omega_{2}\right\}=U$ will be a 2-fundamental system (in $k_{4}$ ) unless one of the following exceptions occurs:

$$
\left( \pm \Omega_{1} \Omega_{2}^{g_{2}} \epsilon^{g_{0}}\right)^{1 / 2} \in k_{4}, \quad\left(g_{i} \in Z\right) .
$$

This is possible only when $e=2, u$ is odd, $v=1$. A further necessary condition is that $\epsilon_{1}$ be a perfect square in $k_{2}$, or, equivalently, $\Omega_{1} / S \Omega_{1}$ be a perfect square in $k_{4}$. The other exception is

$$
\left( \pm \Omega_{2} \epsilon^{g_{0}}\right)^{1 / 2} \in k_{4}, \quad\left(g_{0} \in Z\right) .
$$

This is possible only when $d^{\prime}$ is square-equivalent to $d, 2 d$, or 2 , i.e., $\mathbf{Q}\left(d^{1 / 2}, d^{1 / 2}\right)=$ $\mathbf{Q}\left(d^{1 / 2}, 2^{1 / 2}\right)$.

THEOREM 9.13. For $e= \pm 2$ or $e= \pm 4$ and $p$ odd, the units $\left\{\epsilon, \Omega_{1}, \Omega_{2}\right\}$ will be a p-fundamental system (in $k_{4}$ ) if and only if

$$
U^{*}=\left\{\Omega_{1} / S \Omega_{1}, \Omega_{2}^{*} / S \Omega_{2}^{*}\right\}
$$

is a p-fundamental system (in $k_{4}$ ). 
For Theorem 9.12(i), note that if $\pm \Omega_{1} \Omega_{2}^{g} \epsilon^{g_{0}}=Z^{2}$, then $\left(\Omega_{1} / S \Omega_{1}\right)\left(\Omega_{2} / S \Omega_{2}\right)^{g_{0}}$ $=Z_{0}^{2}$ for some $Z$ and $Z_{0}$ (units) in $k_{4}$. (Note $\epsilon$ disappears.) Thus, by $(9.9 \mathrm{~b}), \Omega_{1} / S \Omega_{1}$ and $\Omega_{1} S \Omega_{1}=\epsilon_{1}$ are perfect squares. The restriction to $e=2, u$ odd and $v=1$ follows from the totally positive nature of $\Omega_{1} / S \Omega_{1}$ (after some minor calculations on the asymptotic behavior of $\xi$ ).

For Theorem 9.12(ii) we refer to (9.9b). If $\pm \Omega_{2} \epsilon^{g_{0}}$ is a perfect square in $k_{4}$ so is $N\left(2 \gamma_{1}\right)=+8$, (in fact +8 and not -8 since $k_{4}$ is totally real).

For Theorem 9.13, note that if $\pm \Omega_{1}^{g_{1}} \Omega_{2}^{g_{2}} \epsilon^{g_{0}}=Z^{p}$ (for $g_{i} \in \mathbf{Z}$ ) then $\left(\Omega_{1} / S \Omega_{1}\right)^{g_{1}}\left(\Omega_{2} / S \Omega_{2}\right)^{g}=Z_{0}^{p}$ for $Z, Z_{0}$ again in $k_{4}$. (The exponent 2 introduced by $\Omega_{2}^{*} / S \Omega_{2}^{*}$ is, of course, immaterial.)

Remark 9.14. Since the search for fundamental units in $k_{4}$ seems to cancel out $\epsilon(=S \epsilon)$ whether or not $\epsilon_{1}$ is fundamental for $k_{2}$, or more relevantly for the ring $\mathbf{Z}[\alpha]$, we need not be concerned that $d$ lies outside the range of standard tables of quadratic units for most cases. The special exceptions in Table III (below) are easily treated by direct decimal comparison of $\epsilon_{1}$ with (say) Ince's table [6], however.

Remark 9.15. To contrast our $p$-adic search for fundamental units with an "archimedean" search (based on absolute values) we should concentrate on $\eta / S \eta$ for $\eta$ any unit of $k_{4}$ (with conjugate $\eta^{\prime}$ ) as in Section 5 (above). Let

$$
\begin{gathered}
L(\eta)=\log |\eta / S \eta|, \quad L\left(\eta^{\prime}\right)=\log \left|\eta^{\prime}\right| S \eta^{\prime} \mid, \\
V(\eta)=\left(L(\eta), L\left(\eta^{\prime}\right)\right), \quad\|V(\eta)\|=\max \left(|L(\eta)|,\left|L\left(\eta^{\prime}\right)\right|\right) .
\end{gathered}
$$

Then the fundamentality of the unit set $U$ in (9.8) reduces to the question of whether or not $V(\eta)$ lies in the lattice $\left[V\left(\Omega_{1}\right), V\left(\Omega_{2}\right)\right]$. If $V(\eta)$ does not, $\eta$ can be replaced (by $\eta \rightarrow \eta \Omega_{1}^{t_{1}} \Omega_{2}^{t_{2}}, t_{i} \in \mathrm{Z}$ ) so that $V(\eta) \neq(0,0)$ but

$$
V(\eta) \leqslant\left(\left\|V\left(\Omega_{1}\right)\right\|+\left\|V\left(\Omega_{2}\right)\right\|\right) / 2=K .
$$

The constant $K$ limits the number of equations for $\eta / S \eta$ to be tested, and thus $K$ limits the index of the unit subgroup $\langle U\rangle$ (and with it, $p$ ). To be practical, we might set $H=\eta / S \eta+S \eta / \eta$ so $H \in k_{2}$. Then the equation for $H$, namely $H^{2}-A_{1} H+A_{2}=0$, would be limited by conditions of discriminant in $k_{2}$, namely $A_{1}^{2}-4 A_{2}=d_{0} m_{1}^{2}$, and in $k_{4}$, namely $\left(4+A_{2}\right)^{2}-4 A_{1}^{2}=d_{0}^{\prime} m_{2}^{2}$. (Here $A_{1}, A_{2}, m_{1}, m_{2} \in \mathbf{Z}$ and $d_{0}, d_{0}^{\prime}$ are the square-free kernels of $d, d^{\prime}$.) Nevertheless, the bounds derived from (9.17) are very large. Even in one of the simplest cases, $e_{1}=-2, a=1, d=17, d^{\prime}=38$, we find $\left|A_{1}\right|<3000,\left|A_{2}\right|<1500^{2}$; and we have many extraneous $H$ to still eliminate.

10. Computational Procedure. We set up a file of primes, usually $p=\{2,3,5,7\}$, for which the case $(e, a, d)$ is being tested. We search for suitable primes $q$ in each case. Such a prime is one for which $q \equiv 1(\bmod 2 p)$ so $p \mid \phi(q)$ and $(-1 / q)=1$ (for convenience), and a further "splitting property" is valid. For this, we require that $q^{\text {odd }} \| f(m)$ for some $m \in \mathbf{Z}$ so $q$ splits completely in $k_{4}$, and at the same time, $\left(d^{\prime} / q\right)=1$ so $q$ splits completely in $k_{8}$. (We take the precaution $q \nmid d d^{\prime}$ so $N \alpha$ and $N \mu$ are prime to $q$.) For such a $q$, the roots $\xi, S \xi, \xi^{\prime}, S \xi^{\prime}$ are congruent to rationals (modulo prime $q \mid q$ in $k_{8}$ ). These rationals can be found directly from (9.4) and 
(9.5) by taking square roots in $\mathbf{Z} / q \mathbf{Z}$ each time. It becomes useful to program an internal subroutine for tables of indices and powers modulo $q$; thus, the square roots are easy to find. (This involves the machine hunting for a primitive root at the same time it constructs the tables.)

In this way $\Omega_{1} / S \Omega_{1}, \Omega_{1}^{\prime} / S \Omega_{1}^{\prime}, \Omega_{2}^{*} / S \Omega_{2}^{*}, \Omega_{2}^{* \prime} / S \Omega_{2}^{* \prime}$ are congruent to rationals from $(9.9 \mathrm{a}, \mathrm{b})$, which are denoted by the same symbols. Thus, using the index function "ind" from our table, we define $D(q) \bmod (q-1)$ as follows:

$$
D(q)=\left|\begin{array}{ll}
\text { ind } \Omega_{1} / S \Omega_{1} & \text { ind } \Omega_{2}^{*} / S \Omega_{2}^{*} \\
\text { ind } \Omega_{1}^{\prime} / S \Omega_{1}^{\prime} & \text { ind } \Omega_{2}^{* \prime} / S \Omega_{2}^{* \prime}
\end{array}\right| \text {. }
$$

Now consider Theorem 9.12i $(p=2)$. Here $\Omega_{2} / S \Omega_{2}$ is always a perfect square so $\Omega_{2}^{*} / S \Omega_{2}^{*}$ was a wiser choice! The case $9.12(\mathrm{i})$ can occur only if $\Omega_{1} / S \Omega_{1}$ is a perfect square or only if $D(q) \equiv 0(\bmod 2)$ for each $q$ chosen. We next compare the determinant to the numerical (regulator) determinant of Section 5 (above). We can apply Theorem 9.13 ( $p$ odd). Here, more simply, $U$ is $p$-fundamental unless $D(q) \equiv 0$ $(\bmod p)$ for each $q$ chosen.

From these tests, we are able to negatively establish the 2 -fundamental nature of the units $U$ for all cases except where $e= \pm 4$. (There are only five such totally real cases to check by hand.) The cases of Theorem 9.12(ii) are checked by examining the square-free kernels of $d$ and $d^{\prime}$. (In fact, the only case to consider here was $(e, a, d)=$ $(-2,-9,113), d^{\prime}=226$, which a simple hand calculation eliminates.) In any case, for $p=2$, we can test for the few configurations where $\pm \Omega_{1}^{g} \Omega_{2}^{g_{2}} \epsilon^{g_{0}}$ is totally positive and take square roots. Unless we can assign signs to the (numerical) square roots which make integral-looking traces, we know the set $U$ is 2 -fundamental.

Otherwise, all $p$-fundamentality assertions were tested by machine in the cases $u \leqslant 15$ in $(9.3 \mathrm{a}, \mathrm{b}, \mathrm{c})$ for $p=2$ and in a subsequent run for $p=3,5,7$. The exceptions are shown in Table III (below).

\section{TABLE III}

$$
\begin{array}{cccccccc}
p & e & a & d & d^{\prime} & \epsilon_{1} & \epsilon & \text { new unit } \\
2 & 4 & 1 & 113 & 4^{2} \cdot 2 & -\epsilon & 776+73 \cdot 113^{1 / 2} & \left(-\epsilon \Omega_{2}\right)^{1 / 2} \\
3 & -4 & 1 & 33 & 2^{2} \cdot 37 & -1 / \epsilon & 23+4 \cdot 33^{1 / 2} & \left(\Omega_{1} \Omega_{2} / \epsilon\right)^{1 / 3} \\
2,3 & -2 & -17 & 353 & 19^{2} \cdot 2 & 1 / \epsilon & 71264+3793 \cdot 353^{1 / 2} & \left(-\Omega_{2}\right)^{1 / 2}\left(\Omega_{1} / \epsilon \Omega_{2}\right)^{1 / 3} \\
2 & 2 & -43 & 9^{2} \cdot 17 & 271 & -1 / \epsilon^{12} & 4+17^{1 / 2} & \left(\epsilon \Omega_{1} \Omega_{2}\right)^{1 / 2}
\end{array}
$$

Illustrations of dyadotropic fields $k_{4}$, which are totally real, (ignoring cases in Table II), and for which the units $U=\left\{\epsilon, \Omega_{1}, \Omega_{2}\right\}$ are not $p$-fundamental (although independent). New units, not generated by $U$, are listed.

11. Machine Program. The file of test primes, and the few cases where $e= \pm 4$ were fed in individually (from a remote terminal), while the cases where $e= \pm 2$ were generated internally in order of increasing $u$, as parametrized in $(9.3 \mathrm{a}, \mathrm{b}, \mathrm{c})$. Thus, the coefficients of $f(x)$ are known. 
The machine successively generates $f(0), f(1), f(-1), f(2), f(-2), \ldots$, keeping a record of $|f(m)|=2^{T_{m}}$ (as an error check on the dyadotropic property). The machine examines for each $m$, the odd $q$ for which $q^{\text {odd }} \| f(m)$, while $q \Varangle d d^{\prime}$. Such a $q$ is retained if $q \equiv 1(\bmod 2 p)$ for some $p$ in the file of test primes for $p$-fundamentality. If $D(q) \neq \equiv(\bmod p)$, then $p$ is dropped from the file (as shown in printouts, see Table IV) and $U$ is declared " $p$-fundamental" for the particular $(e, a, d)$.

If $D(q) \equiv 0(\bmod p)$ for some $p$ and ten values of $q$, the machine "reluctantly" admits that $U$ might not be $p$-fundamental. In any case, the values of $D(q)$ are always printed. (Actually, $|m| \leqslant 100$ is maintained to avoid overflow.) The output of the second case of Table III is indicative. (See Table IV.) Here the file $\{p=3,5,7\}$ was run, and we can see how $p=3$ survives.

$$
\begin{array}{cccccccc}
\multicolumn{1}{c}{\text { TABLE IV }} \\
\multicolumn{7}{c}{e=-4, a=1, d=33, d^{\prime}=2^{2} \cdot 37, p_{i}=\{3,5,7\},} \\
\text { line } & x & f(x) & q & r & q-1 & D(q) & p \\
6 & -25 & 365,116 & 2467 & 2 & 3 \cdot 822 & 3 \cdot 234 & \{3,5,7\} \\
7 & 35 & 1,523,776 & 821 & 2 & 5 \cdot 164 & 5 \cdot 22 & \{3,5,7\} \\
8 & 39 & 2,348,284 & 379 & 2 & 3 \cdot 7 \cdot 18 & 3 \cdot 36 & \{3,5\} \\
9 & -45 & 3,977,296 & 751 & 2 & 3 \cdot 5 \cdot 50 & 3 \cdot 52 & \{3\}
\end{array}
$$

Output used in reducing the file $\{\cdots\}$ of test primes by eliminating $p$ for which $U$ is $p$-fundamental. Here $r$ is the smallest primitive root $(\bmod q)$ from which a table of indices is constructed internally. Lines 8 and 9 eliminate $p=7$ and 5 (respectively), but $p=3$ survives each test (whether or not a factor of $q-1$ ).

For the cases that were not totally real, (actually those in Table II), the calculation still makes sense since $\mu^{1 / 2}$ can exist modulo $q$ even if $\mu<0$ ! This gives us a valuable check on the method, since all $D(q) \equiv 0(\bmod p)$, as we are dealing with necessarily dependent units $\epsilon, \Omega_{1}, \Omega_{2}$ in $U$. Actually, for $e= \pm 4$ there were five cases and for $e= \pm 2$ there were 79 cases with $k_{4}$ totally real. Overflow did not occur in single precision integral arithmetic, (approximately 12 digits).

In two cases, the numerical evidence indicated $U$ might not be 3 -fundamental. The machine computed in a separate run the numerical values of

$$
\left(\Omega_{1} / S \Omega_{1}\right)^{g_{1} / 3}\left(\Omega_{2}^{*} / S \Omega_{2}^{*}\right)^{g_{2} / 3}=Z
$$

as $\left(g_{1}, g_{2}\right)$ takes all pairs modulo 3 . Since $Z$ and $Z^{-1}$ satisfy the same equation, it suffices to find $\left(g_{1}, g_{2}\right)$ which make the traces of $Z$ and $Z^{2}$ integral. Of course, two such pairs occur, $\left(g_{1}, g_{2}\right)$ and $\left(3-g_{1}, 3-g_{2}\right)$, (ignoring the trivial $\left.(0,0)\right)$. The results appear in Table III as new units whose index is a multiple of three. 
Conclusion 11.1. With certainty, when $|e|=4$ and $|e|=2(u \leqslant 15)$, the units $U$ are p-fundamental for $p=2,3,5,7$ except in cases of Table II (dependency) or Table III.

CONJECTURE 11.2. If we modify $U$ by changing $\Omega_{2}$ to the new unit of Table III, then $U$ becomes fundamental for each case tested here.

Clearly, this conjecture can be answered by a finite but (for now) very long computation (see Remark 9.15). The following is more precarious:

CONJECTURE 11.3. For the fields parametrized in Section 9, fundamental units are those listed in the set $U$, except for the cases cited in Tables II and III.

12. Normed Dyadotropics of Various Degrees. The ideas do not trivialize in the quadratic case if we define a dyadotropic quadratic consistently, (as a monic integral quadratic polynomial with three consecutive values which are absolute powers of 2).

THEOREM 12.1. The most general dyadotropic quadratic polynomial has the form: $f_{2}(m \pm x),(m \in \mathbf{Z})$, where

$$
f_{2}(x)=x^{2}+x\left(1-2^{u}(-1)^{v}\right)+2^{u}(-1)^{v}
$$

The ring $\mathrm{Z}[\xi]$ for a root $f_{2}(\xi)=0$ has discriminant

$$
d=1-6(-1)^{v} 2^{u}+2^{2 u}, \quad 0 \leqslant u, v(\bmod 2),
$$

and the readily available unit

$$
\epsilon_{1}=(\xi+1) /(\xi-1)^{u+1}, \quad N \epsilon_{1}=(-1)^{v} .
$$

The proof is left as an exercise, as it follows Theorem 3.5 (above) but in a simpler way. We observe that, again, if $f(m)=(-1)^{S_{m}} 2^{T_{m}}, T_{m}>0$, for $m=-1,0$, 1 then we can normalize so that $T_{1}=1$ and $u=T_{-1}-1$. The first two polynomials $u=0, v= \pm 1$ are

$$
f_{2}(x)=x^{2}-x+2, \quad f_{2}(x)=x^{2}+3 x-2
$$

with $d=-7, d=17$. They represent powers of 2 for an extraordinary range of $x$, i.e., $-2 \leqslant x \leqslant 3,-6 \leqslant x \leqslant 3$ (respectively) so translates of these polynomials reappear several times in the form of (12.2). For $u \leqslant 8$, the units were small enough to check by pocket computer. They are fundamental except for

$$
\begin{gathered}
(u, v)=(3,0), \quad d=113, \quad \epsilon=\epsilon_{1}^{1 / 2}, \\
(u, v)=(5,0), \quad d=833=17 \cdot 49, \quad \epsilon=\epsilon_{1}^{1 / 8} .
\end{gathered}
$$

Actually, the values of $d$ are familiar, as they arise from special cases $[-2 ; u$, $0,0]$ and $[-2 ; u, 1,1]$ of Table I (above) or (9.3b). Also, $\epsilon_{1}$ is precisely the unit in (6.2). Thus, in some sense, biquadratic dyadotropics are related to quadratics.

More generally, we should consider a field $k_{r}$ of degree $r$ with an integer $\alpha$, and $e= \pm 2^{t}(t>0)$. Then set

$$
g_{r}(x)=x^{2}+\alpha x+e, \quad f_{2 r}(x)=N g_{r}(x),
$$

where we form the norm polynomial over all conjugates of $\alpha$ in $k_{r}$. Thus, Part I, [12], 
was concerned with the cases $f=f_{4}$ (where $r=2$, the normed dyadotropic biquadratics). We made the restricting assumption that $d_{0}\left(=\operatorname{disc} k_{2}\right) \equiv 1(\bmod 8)$ for convenience of unit calculation, (e.g., so that $f(1)= \pm 2$ ). There are certainly other cases which we do not attempt to list completely here. A sample case is

$$
f_{4}^{*}(x)=x^{4}-9 x^{2}+4=N\left(x^{2}+5^{1 / 2} x+2\right) .
$$

THEOREM 12.9. The most general normed biquadratic dyadotropic polynomial is $f_{4}(x)=N g_{2}(x)$ with

$$
\begin{gathered}
e=-2 ; \quad \alpha^{2}-(X-Y) \alpha+(X+Y-1)=0, \\
e=+2 ; \quad \alpha^{2}-((X-Y) / 3) \alpha+(X+Y-9)=0
\end{gathered}
$$

and $X, Y$ denoting $\pm 2^{t}, t \geqslant 0$.

The cases where $e= \pm 4$ are those given earlier in (9.3c). No other cases occur (although this is conjectural for $e \neq-4$ ).

Case (12.8) arises with $e=2, X=Y=2$.

The proof is omitted as it is quite mechanical and it is based on (say) these values of $f_{4}(x)=f(x)$ :

$$
\left\{\begin{array}{rccccc}
e & f(-2) & f(-1) & f(0) & f(1) & f(2) \\
-2 & 8 Y & 2 X & 4 & 2 Y & 8 X \\
2 & 8 Y & 2 Y & 4 & 2 X & 8 X
\end{array}\right.
$$

(We note the resemblance of Lemma 3.1 which is more special.)

For the bicubic case $(r=3)$ the dyadotropics resist parametrization. It is simply too hard to make seven consecutive values of $f_{6}(m)$ equal to $( \pm)$ powers of 2 . A manual search seems to lead to only the two following cases $(e=-2)$ :

$$
\begin{gathered}
f_{6}(x)=x^{6}-21 x^{5}+17 x^{4}+113 x^{3}-34 x^{2}-84 x-8 \\
f_{6}(-3 \text { to }+3)=(4096,128,-32,-8,-16,256,-512), \\
\alpha^{3}+21 \alpha^{2}+23 \alpha-29=0
\end{gathered}
$$$$
k_{3}=Q(\alpha), \quad \operatorname{disc} k_{3}=31^{2}, \quad \operatorname{disc} k_{6} / k_{3}=40609,
$$

so $k_{3}$ is abelian; and the other case,

$$
\begin{gathered}
f_{6}(x)=x^{6}+81 x^{5}-271 x^{4}-157 x^{3}+542 x^{2}+324 x-8 \\
f_{6}(-3 \text { to }+3)=(-32768,-4096,16,-8,512,-128,64) \\
\alpha^{3}-81 \alpha^{2}-265 \alpha-167=0
\end{gathered}
$$

$$
k_{3}=\mathbf{Q}(\alpha), \quad \operatorname{disc} k_{3}=31 \cdot 14479, \quad \operatorname{disc} k_{6} / k_{3}=13^{2} \cdot 59 \cdot 83 .
$$

Here disc $k_{6} / k_{3}=N\left(\alpha^{2}+8\right)$ is generated by the radical in the root of $g_{3}(x)=x^{2}+$ $\alpha x-2$. (The factor $13^{2}$ is seen to be nonremovable as 13 has no factor of degree 1 in $k_{3}$.)

These results can certainly not be "explained away", but there seems little pos- 
sibility of parametrizing normed dyadotropics of degree $2 r>4$. The unnormed dyadotropic cubics are, however, readily parametrized (as seen in [13]).

Department of Mathematics

City College of New York

138 Street and Convent Avenue

New York, New York 10031

12. H. COHN, “Dyadotropic polynomials," Math. Comp., v. 30, 1976, pp. 854-862. MR 54 \#273.

13. H. COHN, "Note on dyadotropic cubics," J. Pure Appl. Algebra, v. 13, 1978, pp. 37-40.

14. M. POHST, "Berechnung unabhängiger Einheiten und Klassenzahlen in total reelen algebraischen Zahlkörpern,” Computing, v. 14, 1975, pp. 67-78. MR 52 \#8011. 